UNIVERSIDAD SANTO TOMÁS

FACULTAD DE ADMINISTRACIÓN DE EMPRESAS

\title{
INTERCULTURALIDAD Y ORGANIZACIONES. VENTAJAS LOCALES PARA LA APERTURA A MERCADOS GLOBALES.
}

Trabajo de titulación que se presenta como requisito previo a optar por el grado de:

ADMINISTRADOR DE EMPRESAS

Presentado por:

MARÍA TERESA VALBUENA MELO

XIOMARA SÁNCHEZ TOVAR

HECTOR HORACIO MURCIA CABRA

TUTOR

BOGOTÁ D.C, 30 DE NOVIEMBRE

2018

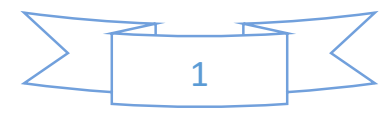




\section{Tabla de Contenido}

1. Resumen 3

2. Palabras Clave y Abstract 3

3. Objetivos 4

3.1. General

3.2. Específicos

4. Introducción 5

5. Marco Teórico 5

6. Metodología, Resultados y Conclusiones 7

6.1. Puerto de Buenos Aires

6.1.1. Interculturalidad en las Organizaciones

6.1.2. Conclusiones

6.2. Puerto de Montevideo

6.2.1. Interculturalidad en las Organizaciones

6.2.2. Conclusiones

6.3. Asociación Latinoamericana de Integración

6.3.1. Interculturalidad en las Organizaciones

6.3.2. Conclusiones

6.4. Super Bold

6.4.1. Interculturalidad en las Organizaciones

6.4.2. Conclusiones

6.5. Capriani

6.5.1. Interculturalidad en las Organizaciones

6.5.2. Conclusiones

6.6. Zona América

6.6.1. Interculturalidad en las Organizaciones

6.6.2. Conclusiones

7. Diario de Campo 17

$\begin{array}{ll}\text { 8. Bibliografía } & 19\end{array}$

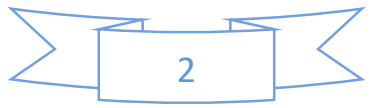




\title{
1. Resumen
}

Se realizó un análisis minucioso a las organizaciones visitadas durante el periplo Argentina y Uruguay, en el cual estas se destacaron ser en su mayoría interculturales y con una visión más abierta al mercado global para la apertura a nuevos mercados internacionales. Son coherentes con sus actividades económicas ayudando así el fortalecimiento y desarrollo económico-social del país en que se encuentren y la posibilidad de brindar trabajo y estabilidad a nuevas personas extranjeras y nacionales que estén interesadas en trabajar allí. Tienen en cuenta así mismo la igualdad para sus colaboradores y el crecimiento organizacional a través del trabajo mutuo; y la forma en la que se llevan a cabo los procedimientos operacionales para el óptimo desempeño en las actividades para así lograr cumplir con los objetivos fijados por la empresa. Las organizaciones visitadas son destacadas por su potencial y desarrollo en su sector económico.

\section{Palabras Clave y Abstract}

Interculturalidad, Mercado Global, Apertura, Organizaciones, fortalecimiento, Económico-social, internacionales, competitividad.

\begin{abstract}
Interculturality, Global Market, Opening, Organizations, Strengthening, Economicsocial, International, Competitiveness.
\end{abstract}




\section{Objetivos}

\subsection{General}

Identificar la estructura organizacional con respecto a la forma en la que se lleva a cabo el manejo de personal extranjero y como estas empresas de desenvuelven en el entorno regional e internacional a través de sus actividades económicas.

\subsection{Específicos}

$\checkmark$ Indagar sobre cada una de las empresas comprobando como estas ejecutan los procesos más efectivos para mantener su personal y como incentivan la comunicación con personas de diferentes países o regiones.

$\checkmark$ Destacar la manera en la que las empresas buscan un reconocimiento más globalizado de sus operaciones y como podrían llegar a expandirse más a través de su capacidad como empresa.

$\checkmark$ Fundamentar la importancia de la apertura global de los mercados latinoamericanos por medio de la industria y al mismo tiempo de la entrada de inmigrantes para el fortalecimiento económico de las empresas pequeñas, medianas y grandes de cada país

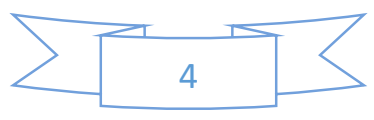




\section{Introducción}

El siguiente informe se justificará el debido análisis a seis organizaciones divididas así, tres de origen Argentino y tres de origen Uruguayo las cuales fueron visitadas por medio de la misión académica periplo internacional de Administración de Empresas que se llevó en cumplimiento en las fechas del 10 de noviembre al 17 de noviembre del 2018.

Las organizaciones fueron analizadas en varios aspectos tales como sociales y económicos, como lo fueron el manejo e impulso de una organización más intercultural y el manejo de las operaciones y proyectos organizaciones, el impulso para la apertura global a nuevos mercados regionales, nacionales e internacionales. Esto con el objetivo final de generar una mayor competitividad en cada una de las organizaciones nombradas las cuales son empresas fuertes y destacadas en su sector económico que se desenvuelven para la generación de propuesta de valor que les de fuerza en su producción y aumento en la rentabilidad.

\section{Marco Teórico}

El presente trabajo está fundamentado partiendo de varios autores exponen en contexto sobre el comportamiento de las organizaciones y por qué el cambio de las mismas son cambiantes según el ambiente.

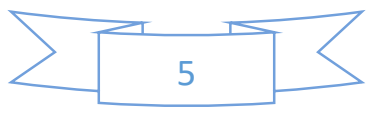


Peter Senge (1992), quizás el autor más conocido en el área, define una organización que aprende, como aquella que expande continuamente su capacidad para construir futuro. Es la integración de talentos y funciones, en una totalidad productiva.

Argyris y Schon (1978), hacen una diferencia entre lo que llaman aprendizaje de un solo ciclo ("single loop learning") y aprendizaje de ciclo doble ("double loop learning"). El primero hace referencia a cambios y correctivos que hacen los trabajadores, con base en las premisas existentes en la organización. El segundo, a cambios que implican establecer nuevas premisas en la organización para superar las actuales, tomándolo como bases por los cambios fuertes que han tenido los trabajadores de Argentina en su economía.

Años después y en la misma dirección, McGill, Slocum y Lei (1992) hablan de aprendizaje adaptativo y generativo. El aprendizaje adaptativo hace referencia a cambios, para facilitar el ajuste de la organización al entorno. Por ejemplo, utilizar know-how para resolver un problema específico, con base en las premisas existentes, que permita continuar con el normal funcionamiento de la entidad. El aprendizaje generativo tiene relación con la transformación del entorno, mediante cambios radicales de estructura, estrategia y sistemas organizacionales. Mientras que el aprendizaje adaptativo busca la acomodación al entorno, el generativo busca transformar el entorno. El aprendizaje adaptativo corresponde al de un solo ciclo y el generativo al de ciclo doble. En uno y otro caso, la organización aprende en su interacción con el entorno.

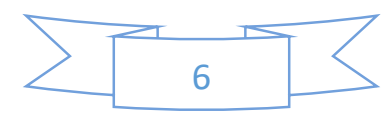




\section{Metodología, Resultados y Conclusiones}

\subsection{Puerto de Buenos Aires}

El puerto de Buenos Aires es uno de los puertos más importantes de Argentina ubicado en la provincia de Buenos Aires con más de 100 años de antigüedad el cual se conecta directamente con el Rio de la plata y da flujo a las embarcaciones que allí se dirigen. Durante la visita se dio la oportunidad de observar el tipo de cargamento entrante y las proporciones de peso en el cargamento en el terminal $\mathrm{N}^{\circ} 5$ operado por la empresa HUTCHISONPORTS BACTSSA.

\subsubsection{Interculturalidad en las Organizaciones}

La visita fue guiada por un colaborador de la empresa encargada del terminal $\mathrm{N}^{\circ}$ 5 en el cual expresaban que había un alto flujo de personas extranjeras puesto que las terminales del puerto eran controladas por compañías portuarias extranjera que son las que se encargan del control total del puerto.

Estas cuentan con al menos un $30 \%$ de sus empleados de otra nacionalidad y el $70 \%$ restante debe ser población del nacional, esto crea diversidad e interculturalidad en el manejo y operación portuaria y así mismo se observa lo que llamaría un contacto entre culturas es un generador de cambio que crea cierta homogeneidad entre las personas y la estructura organizacional de la misma (Antolín, 2015)

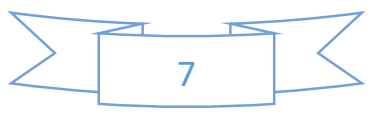


Por lo tanto se puede afirmar que dentro del puerto de Buenos Aires existe un perfecto equilibrio entre la diversidad cultural y la operación en las organizaciones que allí trabajan.

Algunas de las observaciones del puerto que se encontraron llamativas durante nuestra estancia de acuerdo a la apertura global son:

$\checkmark$ El posicionamiento estratégico del puerto que se encuentra en la ciudad principal y más importante de Argentina, esto permite un mayor contacto con las empresas internacionales que allí se ubican y que traen sus materias primas, tecnología y otro tipo de equipamientos para lograr una óptima operación, el constante contacto con estas compañías genera una oportunidad de que más empresas se encuentren interesadas de invertir en el país y así mismo de usar los servicios de las terminales portuarias para importar y exportar sus productos.

$\checkmark$ La integración regional es un componente que les permite lo que bien se llama una profundización en los compromisos en la que hay una liberación comercial (Hiebaum, 2009), también le permiten al puerto tener una mayor apertura y aprovechar mayormente las oportunidades organizaciones para cada una de las terminales que allí operan.

\subsubsection{Conclusiones}

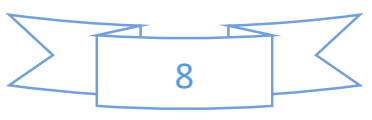


De acuerdo a lo anterior determinamos que el puerto de Buenos Aires es altamente competitivo no solamente por su posicionamiento estratégico, también por su control y administración portuaria, puesto que esto les permite ser tener un mayor abarque en la región. La interculturalidad que existe debido a las empresas que se encargan de operar cada uno de los terminales del puerto permite que haya una mejor conexión y oportunidad de expansión de las empresas para contratar servicios de embarque con otras navieras en otros puertos ya sean cercanos o fuera del territorio nacional.

\subsection{Puerto de Montevideo}

El puerto de Montevideo, Uruguay; el cual se encuentra situado sobre el río de la plata, La Terminal Cuenca del Plata de Montevideo es una instalación que está destinada a la operación de los contenedores. Situado cerca a la bocana del puerto, ha estado en ejercicio desde el año 2002, siendo actualmente la terminal de contenedores más avanzada que hay actualmente en la región.

\subsubsection{Interculturalidad en las Organizaciones}

Dentro del terminal portuario se evita totalmente que se monopolice la actividad del tratamiento de los contenedores que allí ingresan; los operadores traen su personal

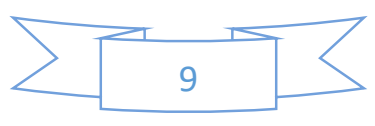


libremente y la operación no es regida por la administración del puerto ya que la operación es totalmente privada. El estado supervisa y controla; esto permite una sana competencia para que los entes externos tengan la posibilidad de escoger entre la diversidad de operadores portuarios que están actualmente.

Esto también incentiva al mejoramiento de la infraestructura del puerto con el fin de brindar una mayor optimización en los procesos de embarque y desembarque; y también en lo que es el cargamento de los vehículos que se llevan lo que están dentro de los contenedores.

Con respecto a la internacionalización que se maneja en la actualidad este puerto al igual que la mayoría cuenta con un $30 \%$ de su personal de operación de otras nacionalidades y el $70 \%$ del país; no cabe duda alguna que esto genera una total ampliación en los mercados de las organizaciones frente a los diversos entornos culturales.

Esto cambia drásticamente las relaciones interpersonales y los obliga a pensar en nuevos métodos para llevar un mejor clima organizacional y también una mejor dinámica, el capital cultural el cual se basa en la integridad, niveles de poder, los aspectos culturales y el respeto (Himmelstern, 2007)

De acuerdo a esto se destacan varios puntos a favor para el puerto de Montevideo, primeramente su ubicación geográfica le permite tener una llegada de los embarques menos costosa y esto lo hace más destacado que sus competidores cercanos por lo cual se hace más opcional para la empresas interesadas en exportar sus productos a países

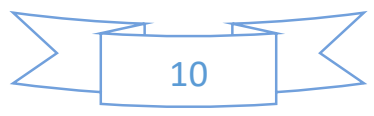


latinoamericanos haciendo su entrada por este puerto, también al ser un país altamente visitado por turistas lo hace muchísimo más llamativo para los cruceros provenientes de Europa lo cual impulsa óptimamente las posibilidades de aumentar el turismo al país y el flujo de cruceros en las temporadas más altas, esto deja en alto el nombre del país y así mismo de las instalaciones del puerto para la recepción de turistas.

\subsubsection{Conclusiones}

Con respecto a lo anterior se puede evidenciar claramente la alta posibilidad que tiene el puerto de Montevideo para seguir manteniéndose y prolongando así las licitaciones que lo mantienen activo dentro del país.

La operación diverso dentro de las instalaciones le permite un mayor movimiento y la inversión para los sitios de embarque y desembarque mejorando la operación general, la interculturalidad de las empresas operadoras en el puerto también es un factor más atractivo puesto que le permite no solamente a las empresas multinacionales que ya se encuentran en el país sino que también a la pequeñas, medianas y grandes empresas que existen actualmente en Uruguay para incluirse también en un mejor desarrollo y globalización de los comodities que allí se producen.

\subsection{Asociación Latinoamericana de Integración}

La asociación latinoamericana de integración (ALADI), es una organización intergubernamental que está compuesta por un total de 13 países los cuales son; Argentina, Bolivia, Brasil, Chile, Colombia, Cuba, Ecuador, México, Panamá, Paraguay, Perú, Uruguay y Venezuela.

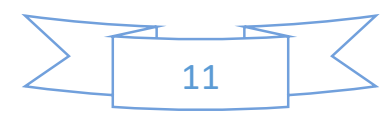


A parte la organización tiene como su principal objetivo la promoción del desarrollo económico-social equilibrado de la región a través de la integración, sus funciones son la promoción y la regulación del comercio mutuo, la complementación económica y la cooperación económica entre los países asociados, también el apoyo a las empresas pymes latinas, ALADI se encuentra ubicada en la ciudad de Montevideo en Uruguay siendo allí su centro de operación, (Integración, 2018).

\subsubsection{Interculturalidad en las Organizaciones}

Esta organización asimila destacablemente la gestión de alianzas para la apertura global para el establecimiento de relaciones comerciales para el beneficio comunitario en la región (Kelly \& David Tanganelli Bernades, 2012), es basada en tres mecanismos; la preferencia arancelaria en la región que es aplicada en los productos producidos en cada uno de los países asociados; los acuerdos de alcance regional y todos los acuerdos de alcance parcial que es llevada a cabo con dos o más países pertenecientes a la región.

Por otra parte esta es una organización altamente multicultural puesto que los representantes de cada país ante la ALDI son mayormente los embajadores de los mismos países asociados, por lo tanto hay un acercamiento más diverso desde la relación entre ellos para el fortalecimiento del comercio en la región.

Así mismo se discuten las proyecciones o proyectos que se llevan a cabo para el aumento en el comercio entre los países asociados y la posible expansión a otros países que aún no conforman la ALADI,

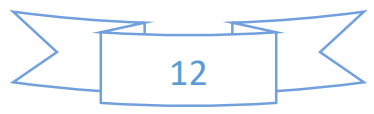


Se tiene actualmente prevista la ingreso de Nicaragua. Esta organización tiene como principios dentro de su conformación el pluralismo, la flexibilidad y la multiplicidad en la forma que concentran los instrumentos comerciales.

\subsubsection{Conclusiones}

A parte es una organización que cumple efectivamente su trabajo; tiene una conformación que la hace multiculturalista y globalizada en su entorno, es dedicada al fortalecimiento del comercio exterior en los países que se encuentran adheridos actualmente y los que se encuentran en este proceso de adhesión, tiene así mismo una alta posibilidad de lograr un mayor abarque global en los países latino americanos añadiendo así a los países que aún no lo conforman para poder crear un mercado más libre y abierto en estos países fortaleciendo la economía y así mismo la calidad de la vida de los habitantes de cada país que lo conforman, para así generar una mayor ventaja competitiva.

\subsection{SuperBol}

Soluciones de Packaging Sustentable es una Pyme familiar ubicada en Buenos Aires, donde se resalta la preocupación que están proyectando por el medio ambiente, siendo consientes desde su fundador hasta su predecesor que es una alta tendencia que debe ser adoptada para continuar en el mercado.

A parte resaltan que lo importante es ser conscientes de nuestros hábitos diarios como consumidores o en sus desempeños laborales. No siendo menos visible el gran

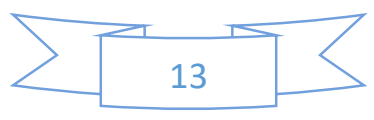


impacto sobre el cual está pasando la economía de Argentina y sobre la cual han tendido a desaparecer varias empresas, y SuperBol ha podido sortear de la mejor manera estos retos.

\subsubsection{Interculturalidad en las Organizaciones}

En esta empresa, si bien maneja empaques para empresas de marcas muy reconocidas en el mercado, se destaca su producto a base de desperdicio de maíz (Amero, caña) para fabricar una bolsa que puede ser biodegradable en 6 meses bajo tierra como lo representa la imagen (Foto 1). Además dentro de la producción ellos marcan su producto para control y referencia de su producción asumiendo también la responsabilidad de que el producto fue conseguido por ellos bajo la máxima calidad y que cualquier desperfecto podrá ser tramitado e identificado con esa referencia. (Figura 2)

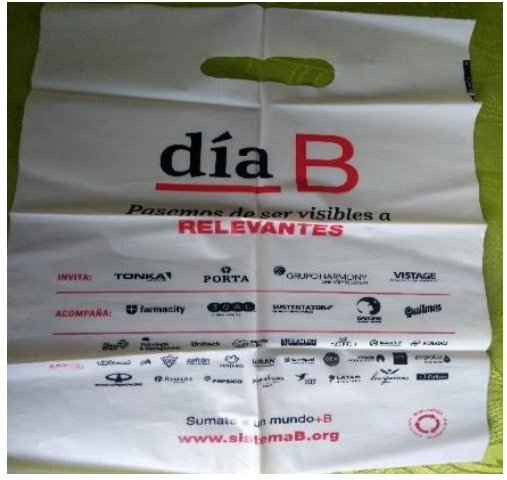

Figura 1

Bolsa con material reciclable

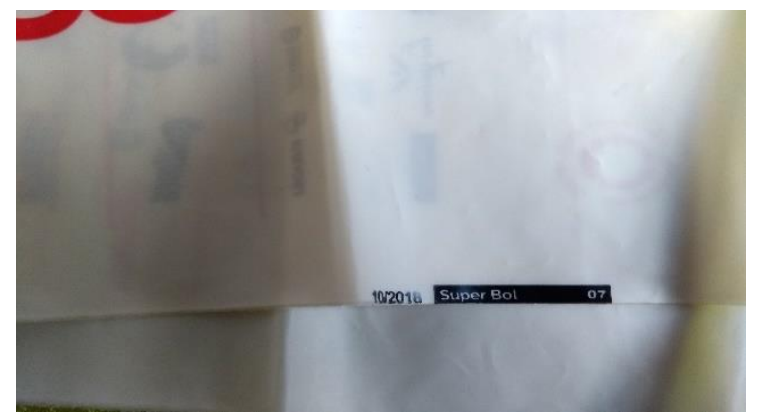

Figura 2

Marca del lote de producción

Tomando como referente a Peter Senge (1992), define una organización que aprende, como aquella que expande continuamente su capacidad para construir futuro,

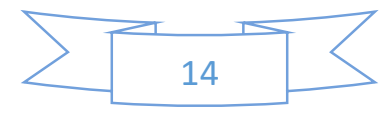


se aplica este criterio para esta empresa, dado que desde el fundador quien empezó con conocimientos heredados por sus padres, y que al llegar el hijo quien pone en práctica lo aprendido en la academia, en la organización logran un complemento para obtener un crecimiento sobre la misma, siendo la integración de talentos y funciones, en una totalidad productiva.

\subsubsection{Conclusiones}

Es una fuerte empresa que ha podido sortear de la mejor manera las crisis de su país, donde la unión hace la fuerza y a pesar de ver un panorama oscuro buscan opciones y nuevas estrategias fortaleciéndolos y generándole un alto crecimiento como entidad.

\subsection{Campari Argentina}

La visita a la multinacional Campari Argentina muestra cómo se puede establecer en un país, conociendo el mercado y la cultura de las personas, manteniendo un legado, son responsable en mercadeo decía que a la segunda mitad de los años noventa, la industria de bebidas se fue caracterizando por una fuerte tendencia de fusiones y adquisiciones comprando a varias empresas lo que lleva así a la creación de corporaciones de alcance mundial y articulaciones notables de la cartera.

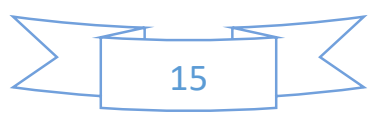


Lo que busca Campari es establecer una bebida, pero con el fin de esta como entrada y que su preparación sea tan perfecta como lo indica la receta para que indique la fluidez y exquisitez que lo caracteriza, Siendo así la razón que pocos como ellos lo dicen lo saben degustar de tal manera.

\subsubsection{Interculturalidad en las Organizaciones}

La organización por ser una multinacional está basada en los lineamientos y valores generados desde su inicio, por sus fundadores y su impacto y reto más grande es lograr educar a las personas sobre la forma de consumo de la bebida.

\subsection{Zonamerica}

Zonamerica busca un total equilibrio Arquitectura + Naturaleza + Servicios que llevan a sus trabajadores a la interacción humana, la conectividad, la diversión, la innovación y la creatividad de fusiones y adquisiciones comprando a varias empresas. Esto conduce a la creación de corporaciones de alcance mundial y coyunturas notables de la cartera, así como lo citan su página web y lo dice textualmente la expositora bajo el recorrido, donde indicaron las empresas que están allí ubicadas y todos los grandes beneficios que estas traen.

\subsubsection{Interculturalidad en las Organizaciones}

Esto es lo más destacado organización en la interculturalidad puesto que hay personas de todos lados aprendiendo y llevando a cabo sus sueños en un mismo lugar.

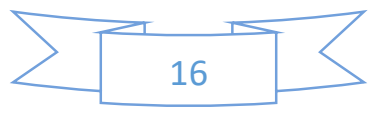


Además, se destaca la base de operaciones de empresas internacionales en el país que lidera el mapa de las localizaciones estratégicas regionales.

\subsubsection{Conclusiones}

Es una entidad con alta expansión en el sector que sabe lo que busca y es proteger al empresario pero primero dar estabilidad al empleador.

\section{Diario de Campo}

La opción de grado del periplo es una oportunidad que brinda la universidad para que los estudiantes de pregrado, tengan como opción una visión de otros países en el manejo de su economía, cultura y además el comportamiento de otras universidades para ampliar la visión de posgrados.

Comienzan a llegar los correos para ir a las reuniones en donde hay que definir el lugar a viajar, donde indican los costos, visitas a empresas a universidades y culturales, las opciones Mexico, Argentina y Peru estas ordenadas del costo más alto al más bajo. Si bien uno de las dudas que se tienen al inicio es si se va a perder el dinero o si este tipo de práctica será provechoso para la vida personal y profesional, o si simplemente valdría la pena hacer el viaje.

Con un grupo de viaje donde unos fueron más escépticos que otros se armó el cupo para ir a Argentina, con el Docente Hector Murcia como nuestro acompañante y Julieth de la agencia Travel Acces. Los cuales nos llevaban a un cumplimiento perfecto de la

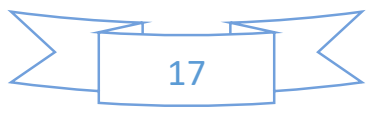


agenda de visitas y traslados además con toda la buena disposición de ejecutar un excelente viaje.

La experiencia llevada en las fechas programadas desde nosotras que fuimos compañeras de habitación y por esto armamos equipo para hacer el presente informe, fue muy grata, en Argentina el hospedaje fue bueno y más por lo centrado que se encontraba pudiendo aprovechar el comercio de la zona y ver el movimiento económico que se mueve hasta altas horas de la madrugada, la hospitalidad de la gente es muy diferente a lo que se ve en Colombia pero durante las visitas a las empresas, culturales y la universidad nos recibían muy bien. Una de las actividades de ocio que disfrutamos mucho fue la del Señor Tango donde tuvimos sin duda alguna la mejor comida, y un show hermoso lleno elegancia, coordinación, resplandor y buena música, simplemente increíble.

Nos dejó como sorpresa la universidad donde nos contaron que las personas de Administración de Empresas no veían materias de Economía o Derecho, y sentimos una gran fortaleza por parte de nuestra universidad donde la formación de un Administrador es integral.

El paso de Argentina a Uruguay fue en Buquebus, donde se puede ver el aprovechamiento de los diferentes medios de transporte para generar diferenciación y un plus para los turistas. Ya instalados allí donde La ubicación del hotel no era tan buena pero los desayunos y las instalaciones eran excelentes, la sorpresa total fue su

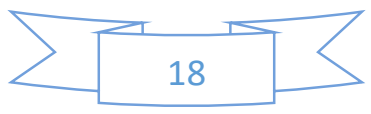


economía y ver el alto costo de las cosas y la comida, los altos impuestos, el valor del transporte.

La atención de las personas era muy bueno, pero porque éramos colombianos denotan mucho una mala energía hacia los argentinos y esto en general. Fue bueno ver a tantas personas en la línea Fit aprovechando las zonas verdes y la playa en grupos haciendo ejercicio.

Fue una experiencia muy buena, si nos preguntaran si es una opción optima de grado la respuesta y sin dudarlo es SI porque siempre hay que ampliar nuestra visión del mundo y esta experiencia es de las que no se repite.

\section{Bibliografía}

Antolín, J. B. (2015). La interculturalidad. Editoria UOC.

Hiebaum, K. (2009). Internacionalización mediante la globalización: el regionalismo y su apertura a la internacionalización. El Cid Editor.

Himmelstern, F. (2007). Las organizaciones de hoy son multiculturales. Revista Javeriana, revistas.javeriana.edu.co/index.php/signoypensamiento/article/view/3713/3378.

Integración, A. I. (27 de Noviembre de 2018). Asociación latinoamericana de Integración. Obtenido de Asociación latinoamericana de Integración: http://www.aladi.org/sitioAladi/index.html

Kelly, M., \& David Tanganelli Bernades, J. L. (2012). Gestion de alianzas estrategicas: Construyendo alianzas que funcionen. Ediciones piramide.

Zonaamerica https://web.zonamerica.com/ recuperado el 28/11/201 